\title{
Highly Reflecting Stable White Paint for the Detection of Ultraviolet and Visible Radiations
}

\author{
J. B. Schutt, J. F. Arens, C. M. Shai, and E. Stromberg
}

\begin{abstract}
The use of polyvinyl alcohol polymer as a binder for barium sulfate does not allow the intrinsically high reflectance of barium sulfate in the 200-300-nm range to be optimally employed. In an effort to better utilize this property, totally inorganic coating systems are described. Potassium sulfate turns out to be the preferred binder for reflectance enhancement. Compositions, formulating procedures, and application techniques are included. For completeness, absolute and relative reflectance data are included for intrasystem and intersystem comparisons.
\end{abstract}

\section{Introduction}

In a previous paper of this series, ${ }^{1}$ the preparation and optical properties of a barium sulfate-polyvinyl alcohol coating were presented in detail. Although providing less reflectance in the near uv and ir spectral regions than freshly smoked magnesium oxide, this coating exhibits greater mechanical strength and has demonstrated improved resistance toward atmospheric aging. For most applications, it has turned out that this compromise is worthwhile. The total reflectance of a coating obtained by weighting it with respect to the extraterrestrial solar irradiance is not dependent in a crucial way upon a highly reflecting coating below $295 \mathrm{~nm}$ because less than $4 \%$ of the solar energy lies below this wavelength. As for the NIR, barium sulfate powder-irrespective of binderis not white reflecting, because its surfaces are polar and absorb water, thus giving rise to a series of adsorption bands. ${ }^{2}$ The loss of white reflectance in the NIR limits its use to reasonably intense sources such as those used in reflectance spectrometers. As a result, the use of barium sulfate powder-with any binder whatsoever-must be restricted at low photon levels to the visible and the near and middle uv regions of the spectrum.

For some unusual applications, however, the low uv reflectance of barium sulfate polyvinyl alcohol relative to smoked $\mathrm{MgO}$ is undesirable. One of these applications involves the collection of light by a chamber designed to detect Cerenkov radiation. To perform this function most efficiently, a coating,

E. Stromberg is with the Chemistry Department of Rhode Island University; the other authors are with Goddard Space Flight Center, Greenbelt, Maryland 20771.

Received 3 April 1974. aside from reflecting electromagnetic energy dominantly in the diffuse mode, must possess the highest possible reflectance over that range of wavelengths produced by the Cerenkov process. This range includes wavelengths from $200 \mathrm{~nm}$ to $800 \mathrm{~nm}$ for the case of a gas filled chamber. To meet the requirement of high reflectance in the neighborhood of 200 $\mathrm{nm}$, it was judged that since the reflectance of high purity barium sulfate powder is comparable to smoked $\mathrm{MgO}$, it should be used as the scatterer. Data given in the previous paper show that with polyvinyl alcohol as a binder barium sulfate does not approach its intrinsic reflectance in this region. From experience, it is known that no organic binder should be present in a coating whose reflectance at about $200 \mathrm{~nm}$ is expected to compare favorably with the virgin powder.

In seeking out an optimum composition, two approaches come to mind. First, one can utilize the concept of differential solvent-solute vapor pressures to deposit barium sulfate. This method is used successfully for sodium chloride; briefly, it involves the dispersion of sodium chloride in a system of liquids of differing vapor pressures at room temperature so that (1) a minimal amount of salt is dissolved, (2) dry spraying is eliminated, and (3) a considerable amount of ionic diffusion is permitted during evaporation of liquids to permit a partially amorphous salt structure to establish itself.

Second, one can utilize the concept of differential solubility. This concept mirrors the former in that a single solvent-dispersion medium is used wherein a material like the high purity barium sulfate with a polar surface is dispersed in, e.g., water along with a more soluble salt. In this case (1) the solubility of one ingredient is required, (2) dry spray is minimal because the salts have negative free energies of hy- 
dration, and (3) the soluble salt crystallizes in the interstices between the barium sulfate particles providing a binding action that inhibits the self-crystallization of the binder and thus forms a heterogeneous matrix. Both types of coatings require primer systems because of their corrosive actions on commonly used metal substrates. The second system is preferably applied over a specially chosen primer, since additional mechanical strength is acquired by the reflective coating from the primer system.

In the next section, compositions are given for the coatings systems as well as methods for formulating and applying them. Subsequently, the chemistry of the paints is presented followed by a section providing optical data for comparisons with other systems.

\section{Coating Development}

\section{Prime Coat}

To obtain a suitable priming coating with maximum reflectance in the uv, a mixture was formed consisting of high purity aluminum oxide and a potassium silicate solution of composition $\left[0.35\left(\mathrm{~K}_{2} \mathrm{O}\right.\right.$. $\left.\left.3.3 \mathrm{SiO}_{2}\right)_{\mathrm{MOLE}}+0.65 \mathrm{H}_{2} \mathrm{O}\right]_{\mathrm{WT}}$ with sufficient additional water to allow the ingredients to be stirred together with a magnetic stirrer until alkaline hydration of the oxide was completed. At this stage, two sets of glass slides were flow coated with the mixture and dehydrated, one under ambient conditions for 24 $\mathrm{h}$ and the other at $100^{\circ} \mathrm{C}$ for $1 \mathrm{~h}$. After each treatment, absolute reflectance measurements were made. It was judged from these data that the ratio sequence of $\left(\mathrm{K}_{2} \mathrm{O} \cdot 3.3 \mathrm{SiO}_{2}\right): \mathrm{Al}_{2} \mathrm{O}_{3}: \mathrm{H}_{2} \mathrm{O}: 1: 3.3: 6.6$ was most satisfactory.

\section{Reflective Coating}

Mixtures of $\left(\mathrm{NH}_{4}\right)_{2} \mathrm{SO}_{4},-\mathrm{BaSO}_{4}$, and water, as well as a saturated solution of $\mathrm{K}_{2} \mathrm{SO}_{4}$ and $\mathrm{BaSO}_{4}$, were flow coated onto the optimum prime coat. Samples were cured according to the schedule used for the prime coat. Baking showed that as the total thickness of the coatings approached about $0.75-0.120 \mathrm{~mm}$ in thickness that samples became subject to bubbling and cracking. The optimum $\left(\mathrm{NH}_{4}\right)_{2} \mathrm{SO}_{4}: \mathrm{BaSO}_{4}$ ratio was determined to be $1: 6$ by reflectance measurements; the system of $\mathrm{BaSO}_{4}$ with $\mathrm{K}_{2} \mathrm{SO}_{4}$ as the binder was found to give the greatest uv reflectance and was therefore judged to have the greatest potential for the detection of Cerenkov radiation in a high performance chamber.

\section{Paint Preparation}

\section{Prime Coat}

To 35 parts of potassium silicate solution $\left[0.35\left(\mathrm{~K}_{2} \mathrm{O} \cdot 3.3 \mathrm{SiO}_{2}\right)_{\mathrm{MOLE}}+0.65 \mathrm{H}_{2} \mathrm{O}\right]_{\mathrm{WT}}, 105$ parts of $\mathrm{Al}_{2} \mathrm{O}_{3}$ and 153 parts of water are added. The resulting mixture is then charged into a jar mill and milled for $1 \mathrm{~h}$. The milled slurry is then applied by spraying until a dry thickness of about $0.2 \mathrm{~mm}$ is obtained (about $0.4 \mathrm{~mm}$ wet) and allowed to air cure at least $24 \mathrm{~h}$ before top coating. Air cure over a period of several hours is required to enable a good adhesive bond to develop between primer and substrate.

\section{Reflective Coatings}

Compositions optimized according to physical and optical characteristics are given in Table I. Systems with $\left(\mathrm{NH}_{4}\right)_{2} \mathrm{SO}_{4},\left(\mathrm{NH}_{4}\right)_{2} \mathrm{SO}_{4}$ and $\mathrm{K}_{2} \mathrm{SO}_{4}$, and $\mathrm{K}_{2} \mathrm{SO}_{4}$ are given for the sake of completeness. $\mathrm{K}_{2} \mathrm{SO}_{4}$ alone gives the highest uv reflectance.

These compositions may be prepared by adding the soluble sulfate(s) to the required amount of water and stirring until a solution is obtained. Finally the $\mathrm{BaSO}_{4}$ is added slowly and stirred until the composition is agglomerate free. Extreme care must be taken in mixing these ingredients together into the solution-slurry combination, because all contaminants will decrease the reflectance over at least a portion of the 200-300-nm band, with the effect being most noticeable in the neighborhood of $200 \mathrm{~nm}$. Particularly harmful are trace amounts of metallic ions comprising the first, second, and third transition metal series in the Periodic Table as well as heavy metals such as lead. (For this reason, the barium sulfate used in this work was purchased from the Distillation Products Division of the Eastman Kodak Company under a product reference nomenclature of White Reflectance Standard. The potassium sulfate employed need only meet ACS Standards for purity.) Mixing must, as a result, be carried out in a Pyrex container employing a glass coated magnet for stirring. The container should be covered and stirring terminated as soon as the barium sulfate is dispersed, a process completed in $1 \mathrm{~h}$ or so for about $100 \mathrm{~g}$ of pigment.

The coating is applied by spraying in one application if possible until a dry thickness of $0.2-0.75 \mathrm{~mm}$ is obtained depending upon application For efficient uv scattering a nominal thickness of $0.2 \mathrm{~mm}$ is more than sufficient; however, for a reflectance maximum through to $2400 \mathrm{~nm}$, thicknesses to $0.75 \mathrm{~mm}$ may be required.

\section{Chemical Aspects}

The optimum coating composition and the one likely to be most often utilized will be $\mathrm{BaSO}_{4}$ dispersed in a $1 \%$ solution of $\mathrm{K}_{2} \mathrm{SO}_{4}$ as shown in Table I. However, because the composition has an acidic $\mathrm{pH}$, care must be exercised in its use, especially since the prime coat is porous. A saturated solution of $\mathrm{K}_{2} \mathrm{SO}_{4}$ has a pH of 5.8. Slurrying $\mathrm{BaSO}_{4}$ at a level of 72 parts in 108 parts of water gives nominally a $\mathrm{pH}$ of 4.2. Combining the ingredients yields a $\mathrm{pH}$ whose value decreased with time from 4.7 to a minimum of 3.5 over a period of several days. If $\mathrm{NaCl}$ is substituted for $\mathrm{K}_{2} \mathrm{SO}_{4}$ on a mole basis, a pH of 2.3 results at equilibrium. When coatings containing $\mathrm{NaCl}$ were applied to aluminum or iron protected by alkaline primer, it was noted that a percolation of aluminum metal or iron hydroxide to the surface of the reflective coating became visually detectable within 24 $\mathrm{h}$. This indicated that the equilibrium $\mathrm{pH}$ of 2.3 for the coating containing $\mathrm{NaCl}$ was too low. 
Briefly, what is occurring in the reflective coating slurry is the exchange of protons and monovalent cations between $\mathrm{BaSO}_{4}$ and solution. Schematically an equilibrium such as

$$
-\mathrm{BaHSO}_{4} \rightleftharpoons-\mathrm{BaSO}_{4}^{-}+\mathrm{H}^{+}
$$

is established, where $-\mathrm{Ba}$ represents a surface barium ion. It follows from the $\mathrm{pH}$ data that when the solution contains $\mathrm{K}^{+}$ions

$$
-\mathrm{BaHSO}_{4}+\mathrm{K}^{+} \rightleftharpoons-\mathrm{BaKSO}_{4}+\mathrm{H}^{+} \text {, }
$$

the equilibrium is shifted to the right side of the equation. The resulting $\mathrm{pH}$ for the coating composition is then dependent upon the ionization constant

$$
\mathrm{HSO}_{4} \rightleftharpoons \mathrm{H}^{+}+\mathrm{SO}_{4}^{-}
$$

as well, and in the case of sodium chloride

$$
\mathrm{HCl} \rightleftharpoons \mathrm{H}^{+}+\mathrm{Cl}^{-}
$$

For $\mathrm{HSO}_{4}^{-}, \mathrm{K}_{a} \sim 10^{-2}$, whereas for $\mathrm{HCl}, \mathrm{K}_{a} \sim \mathrm{O}(1)$. This simple scheme explains the $\mathrm{pH}$ observations for the particular concentrations of ingredients chosen for the measurements.

The primer on the other hand is an alkaline composition of $\mathrm{pH} \simeq 12$. For the composite system, therefore, we need to address ourselves to an acidbase system comprising a rather complex system of potassium salts of barium sulfate, silicic acid, and to a lesser extent aluminum oxide. Since the primer is water sensitive, application of the reflective coating with its lower $\mathrm{pH}$ causes the silicic acid and aluminum oxide potassium salts to discharge by releasing potassium ions in exchange for protons. By the common ion effect it follows that potassium sulfate will deionize and precipitate primarily in the interstices of the primer as well as onto $\mathrm{BaSO}_{4}$. Hence, the alkalinity of the prime coat serves to prevent the growth of potassium sulfate crystals. The common ion effects derived from the dual presence of sulfate and potassium ions give intracoating and intercoating reactions, respectively, which serve to provide enhanced mechanical properties.

\section{Results}

Reflectance data for coatings discussed in previous sections are shown in graphic form in Figs. 1-3. Figure 1 shows reflectances for $\mathrm{BaSO}_{4}$ coatings relative to $\mathrm{BaSO}_{4}$ powder employing both potassium and ammonium sulfate singly and in mixture as binders.

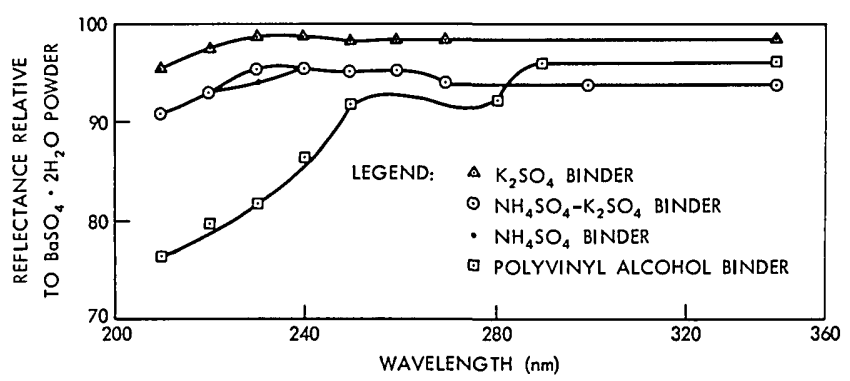

Fig. 1. Ultraviolet reflectance of barium sulfate coatings employing both organic and inorganic binders.

Data for the polyvinyl chloride (PVA) paint have been included for reference. Figure 2 compares five highly reflecting coatings now in use: barium sulfate-polyvinyl alcohol (MS-125), the forerunner system developed by Eastman Kodak, smoked MgO, sodium chloride, and the potassium sulfate system described here over the alkaline primer. Figure 3, included for reference, compares polyvinyl alcohol powder, PVA paint (MS-125), high silica microballoons, and millipore filter paper type $\mathrm{EA}$ to $\mathrm{BaSO}_{4}$.

\section{Comments}

In offering a highly reflecting paint employing barium sulfate as the scatterer and in utilizing an inorganic binder (e.g., $\mathrm{K}_{2} \mathrm{SO}_{4}$ ), rather than an organic one (e.g., polyvinyl alcohol), the reflectance has been improved down to about $200 \mathrm{~nm}$, and improvement is also seen in the coating's aging characteristics as measured by reflectance changes in the uv. From Fig. 3 PVA is seen to have significant uv absorption compared to barium sulfate. Potassium sulfate (not shown), low in transition metal ion concentrations, will have significantly less absorption. PVA can also react slowly with barium sulfate by esterification; such is not the case with potassium sulfate. Under essentially ambient conditions degradation of reflectance did not appear to be a problem for the compositions listed in Table I. Each system, formulated using Eastman Kodak's white reflectance standard barium sulfate, was stored under dry nitrogen or desiccated air. No special illumination conditions were provided. The reflectances of all samples were mon-

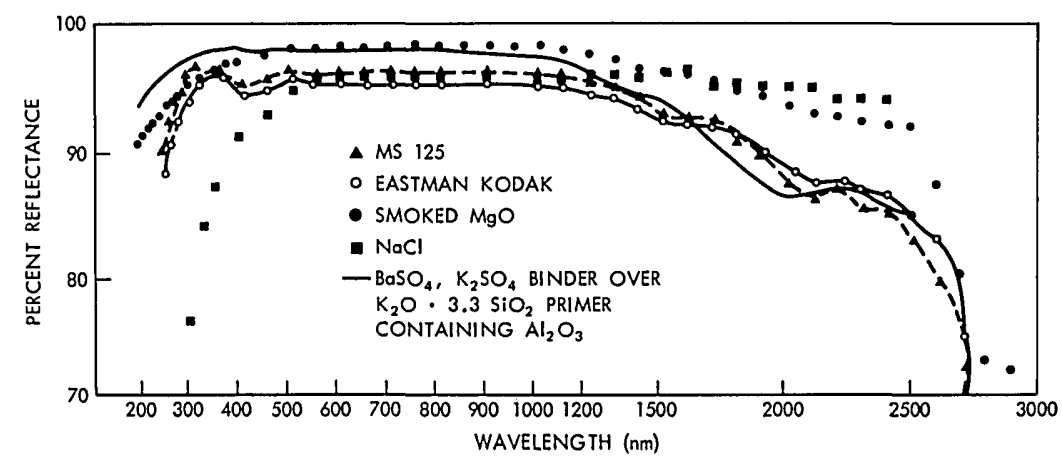

Fig. 2. Comparisons of spectral reflectance data for $\mathrm{MgO}, \mathrm{MS}-125$, Eastman white reflectance standard, $\mathrm{NaCl}$, and $\mathrm{BaSO}_{4}-\mathrm{K}_{2} \mathrm{SO}_{4}$. 


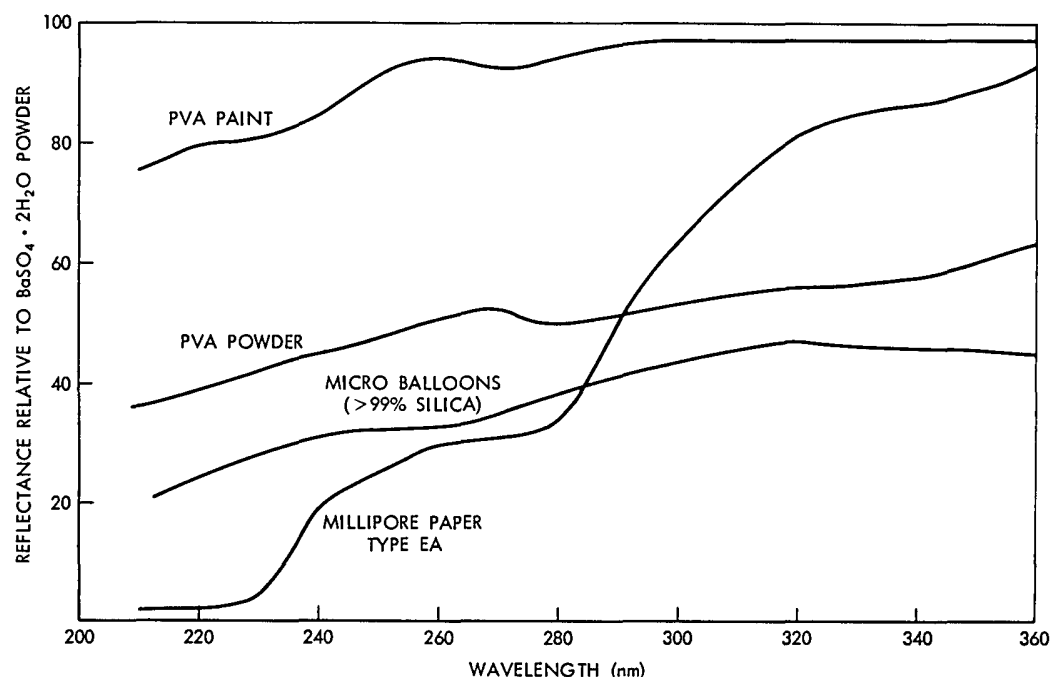

Fig. 3. Curves showing relative uv reflectances of some candidate Cerenkov light reflecting materials.

Table I. Compositions of $\mathrm{BaSO}_{4}$ Coatings Using Sulfates with Monovalent Cations as Binders

\begin{tabular}{|c|c|c|c|c|c|c|c|c|}
\hline \multirow[b]{3}{*}{$\begin{array}{l}\text { Coatings by } \\
\text { binder }\end{array}$} & \multicolumn{8}{|c|}{ Compositions } \\
\hline & \multicolumn{2}{|c|}{$\left(\mathrm{NH}_{4}\right)_{2} \mathrm{SO}_{4}$} & \multicolumn{2}{|c|}{$\mathrm{K}_{2} \mathrm{SO}_{4}$} & \multicolumn{2}{|c|}{$\mathrm{BaSO}_{4}$} & \multicolumn{2}{|c|}{$\mathrm{H}_{2} \mathrm{O}$} \\
\hline & $\begin{array}{c}\text { Parts by } \\
\text { wt. }\end{array}$ & $\%$ & $\begin{array}{c}\text { Parts by } \\
\text { wt. }\end{array}$ & $\%$ & $\begin{array}{c}\text { Parts by } \\
\text { wt. }\end{array}$ & $\%$ & $\begin{array}{c}\text { Parts by } \\
\text { wt. }\end{array}$ & $\%$ \\
\hline Ammonium & 12.0 & 6.25 & - & - & 72 & 37.5 & 108 & 56.2 \\
\hline sulfate & 14.4 & 7.4 & - & - & 72 & 37.0 & 108 & 55.5 \\
\hline binder & 1.01 & 0.7 & - & 一 & 72 & 39.7 & 108 & 59.6 \\
\hline & 1.01 & 0.5 & - & - & 72 & 35.5 & 130 & 64.0 \\
\hline Potassium & 一 & - & 0.009 & Trace & 72 & 40.0 & 108 & 60.0 \\
\hline sulfate & 一 & - & 1.014 & 0.56 & 72 & 39.7 & 108 & 59.6 \\
\hline binder & - & - & 1.014 & 0.5 & 72 & 35.5 & 130 & 64.0 \\
\hline Mixed binder: & 14.0 & 7.4 & 0.009 & Trace & 72 & 37.0 & 108 & 55.5 \\
\hline ammonium & 12.0 & 6.2 & 1.01 & 0.5 & 72 & 37.3 & 108 & 56.0 \\
\hline sulfate, & 1.0 & 0.55 & 1.01 & 0.54 & 72 & 39.5 & 108 & 59.4 \\
\hline $\begin{array}{l}\text { potassium } \\
\text { sulfate }\end{array}$ & 12.0 & 5.2 & 1.01 & 0.49 & 72 & 30.6 & 150 & 63.7 \\
\hline \multicolumn{9}{|l|}{$\mathrm{BaSO}_{4}$} \\
\hline $\mathrm{H}_{2} \mathrm{O}$ & & & & & & & & \\
\hline
\end{tabular}

itored monthly for 6 months by means of a Beckman DK-2A spectroreflectometer. Spectral variations in all cases were found not to exceed about $1 \%$, the level normally considered the measurement accuracy of the instrument. On the other hand, the inorganic coating is more acidic than the mixed system, creating the problem of discoloration by corrosion. The alkaline primer solves the problem nicely provided the reflective coating is freshly prepared.

As with all highly reflecting coatings, care must be continually exercised to keep them free from contaminants arising from all conceivable sources. As the uv reflectance is extended further into the near vacuum uv, contamination becomes more of a problem since most substances will degrade the performance of such a coating. The described paint is highly reflect- ing; ingredients admixed from other judicious choices in the periodic table will not improve reflectance further since the number of combinations is limited in which the electronegativity differences between cationic and anionic species are large.

This work was supported by the High Energy Astrophysical Observatory Project (HEAO).

\section{References}

1. J. B. Schutt and C. M. Shal, "Formulation Procedure and Spectral Data for a Highly Reflecting Coating from $200 \mathrm{~nm}$ to 2300 nm, "Internal Communication X-762-71-266 (1971), NASA Goddard Space Flight Center, Greenbelt, Maryland 20771.

2. L. Morren, G. Vandermaersch, and P. Antoine, Lighting Res. Technol. 4, 243 (1972). 